\title{
Applying deep learning in automatic and rapid measurement of lattice spacings in HRTEM images
}

\author{
Xiaoyang Zhu ${ }^{1}$, Zuoheng Zhang ${ }^{1}, \mathrm{Yu} \mathrm{Mao}^{1}, \mathrm{Yan} \mathrm{Li}^{1}$, Xiao Huang ${ }^{2}$ and Ning $\mathrm{Gu}^{{ }^{1 *}}$
}

Deep learning has been playing increasingly important roles in image processing analysis. The advancement of materials science and engineering has driven the rapid development of transmission electron microscopy (TEM) and related techniques. Efficiently and accurately analyzing TEM data, which are usually in the form of image sequences, has become increasingly important. As a consequence, the past decade has witnessed the continuous innovation and development of methods for processing TEM images. Examples include the high-level identification of atomic columns based on convolutional neural network (CNN) [1], atomic positioning method in high-resolution TEM (HRTEM) images [2] and automatic identification and extraction of nanoparticles in HRTEM images [3].

The fast Fourier transform (FFT) of a TEM image converts the image into an infinite sine wave set with different frequencies, amplitudes and phases, which constitute an important intermediate data set in the image analysis process. When a TEM image contains lattice fringes, its FFT image will show a dot pattern to reflect the lattice regularity [4]. Unfortunately, extracting the regular pattern in an FFT image for lattice analysis and regenerating high-quality lattice patterns usually require manual selection of bright FFT spots, which is time consuming and sometimes can cause un-wanted regions to be selected [5]. Recently, $\mathrm{CNN}$ and related deep learning methods have shown excellent performance in visual recognition tasks [6]. In the process of deep learning, the neural network can be regarded as a function with unknown parameters and relationships. Deep learning with training samples and pre-set standard outputs refines the parameters and functions, so that the output data are within the specified error range from the standards [7]. In the subsequent process, a newly entered input can therefore be predicted by the optimized function. There are many types of deep learning methods, such as CNN [1], recurrent neural networks (RNN) [8], and generative adversarial network (GAN) [9].

Although some deep learning-based methods have been used previously for TEM image analysis [1-3], they have not been used for automatic measurement of lattice spacings in HRTEM images. In this contribution, we applied a U-net training model for automatic extraction of bright regions in FFT images. A new algorithm based on computer vision was also developed for separation of multiple pairs of bright spots in individual FFT images, and measurement of lattice spacings and inter-plane angles. With the provision of standard powder diffraction file (PDF) for known crystals, our method can be potentially applied to analyze TEM images of various crystalline materials. As a demonstration, our procedure was applied to analyze TEM images of magnetic $\mathrm{Fe}_{3} \mathrm{O}_{4}$ nanocrystals.

Fig. 1 shows the workflow of the program. A typical TEM image with regions showing lattice fringes is the input, and the lattice spacing and inter-plane angle measurement results obtained by processing the FFT of the image will be the output. The program consists of two parts, including the automatic measurement of lattice spacing and inter-plane angle (part a), and the comparison of the measured spacing and angle with standard crystal database (part b). The first part can be further subdivided into the deep learning part which is to identify the bright spots in an FFT image (part a1), and the computer vision part which is to determine the lattice spacings and inter-plane angles (part a2).

In more detail, the program will first perform FFT on any TEM image in the jpg format by using discrete Fourier transform (DFT) to represent the finite sum of

\footnotetext{
${ }^{1}$ State Key Laboratory of Bioelectronics, Jiangsu Key Laboratory for Biomaterials and Devices, School of Biological Science and Medical Engineering, Southeast University, Nanjing 210009, China

${ }^{2}$ Institute of Advanced Materials, Nanjing Tech University, Nanjing 211816, China

${ }^{*}$ Corresponding author (email: guning@seu.edu.cn)
} 


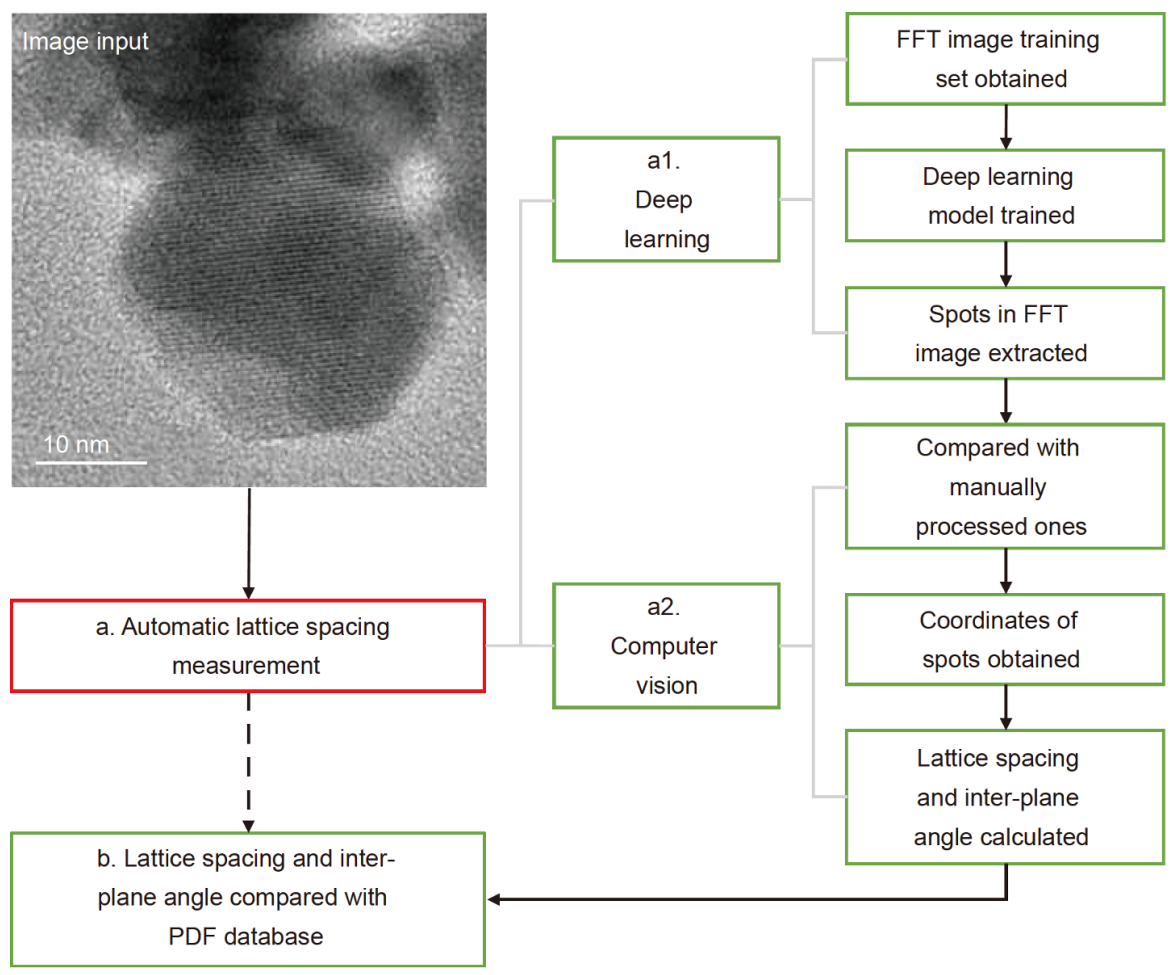

Figure 1 The workflow of the program.

sine functions in order to analyze the discrete signals. In order to train the neural network based on deep learning, 100 FFT images were then manually processed, whose bright diffraction spot areas were covered by white circles with a diameter of 5 pixels using a conventional image processing software (e.g., Adobe Photoshop). The rest area, including the center bright area, was set as black. The original 100 FFT images and their corresponding manually processed images were then used as the training set for subsequent deep learning (Fig. S1). The architecture of the neural network, i.e., the U-net structure employed in the present study, is based on the fully convolutional neural networks (FCNN) [10,11], which ensures that any image with arbitrary dimensions can be processed and every pixel in the image will be analyzed.

Through deep learning with the abovementioned 100 pairs of sample FFT images (each pair of images include an original FFT image and its corresponding image with manually selected bright spots), a processing mechanism was repeatedly optimized and then embedded in the neural network structure to ensure that any arbitrary FFT image processed through the neural network can yield an image similar to the manually processed one. During our deep learning process, the different processing structures (including max-pooling [12], convolution, dropout, de- convolution) [13] in the neural network structure shown in Fig. 2 were continuously improved with the support of a large amount of training data, and after 2000 times of model learning process, a neural network that can be used for extraction of diffraction spots in an FFT image was finally obtained. This means that when a new FFT image is entered to the network as the input, an image with extracted spots will be the output. The detailed description of the deep learning procedure is available in the Supplementary information.

After the bright spots was extracted through deep learning, computer vision was used for the next operations. To evaluate the accuracy of the extraction results, processed images were further compared with manually processed ones. However, since the proportion of the bright spots in a target image is too small, even a completely dark image can achieve an accuracy of over $99 \%$. Therefore, a processed image with predicted spots was firstly subjected to a $5 \times 5$ swell operation and then multiplied with its original FFT image in every pixel coordinate through an "AND operation". If the resulting image also shows white spots with the same coordinates as those in the predicted image, the prediction is correct, and vice versa. Fig. $3 \mathrm{a}$ and $\mathrm{b}$ show examples of the evaluation of the results of predicted images with two and 


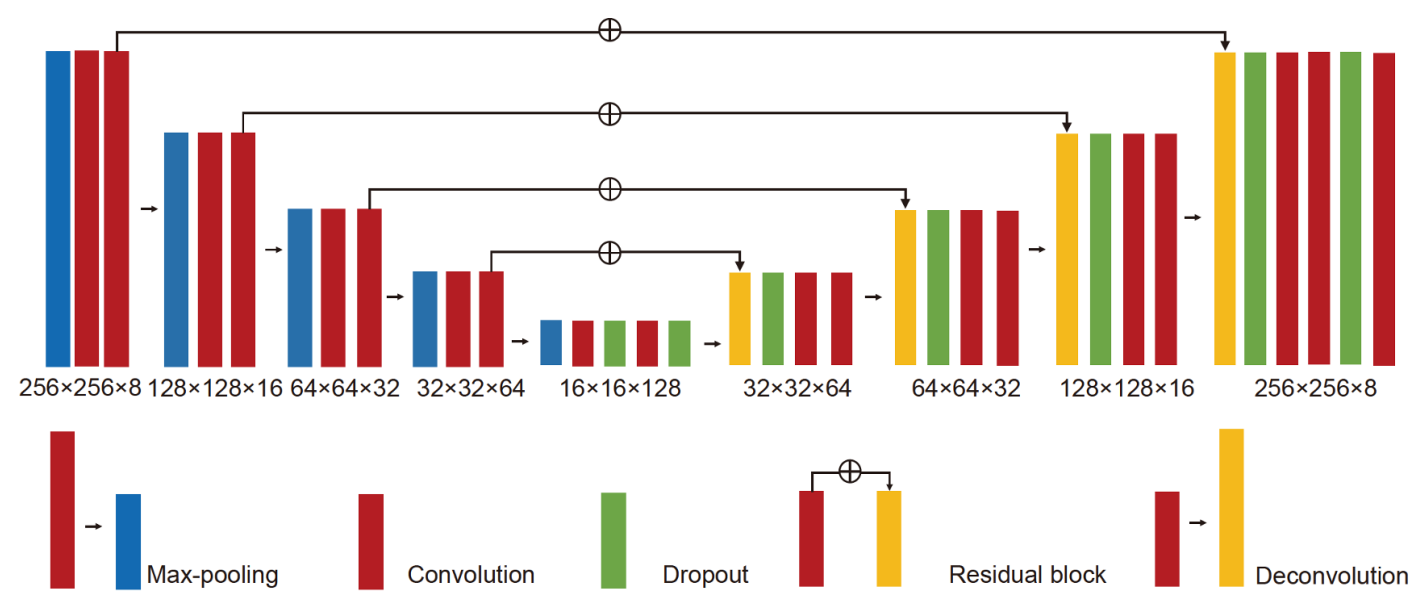

Figure 2 U-net structured neural network architecture with information flowing from left to right.

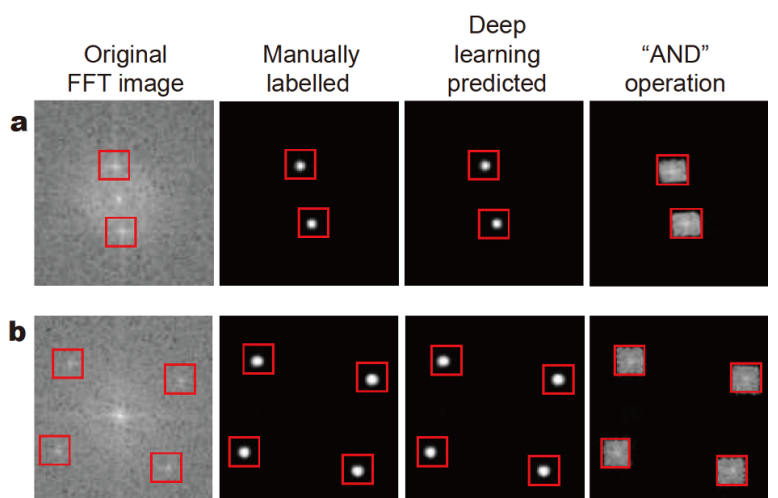

Figure 3 Verification of results predicted by the deep learning model using the FFT image with (a) one pair of bright spots, and (b) two pairs of bright spots.

four spots, respectively.

Note that sometimes, more than one pair of spots are present in an FFT image, and therefore, in order to locate the center point of the image and identify centrosymmetric spots, a recursive function was written to combine the coordinates of pixels belonging to the same white area and determine their average coordinate. Typically, the coordinates of all white pixels (whose RGB value is 255) in a predicted FFT image are collected in a set, defined as $W p$. Given that the top-left corner of an image is set as the origin with coordinate of $(0,0)$ by default, the collected coordinates are arranged in an order from small to large coordinates, i.e., from the left to right and from top to bottom of the image. Then, the coordinates of pixels belonging to a single white spot are grouped into a subset $L_{i}$, where the variable $i$ represents the order of that particular white spot in the image. In each sub-set $L_{i}$, starting with any pixel $\left(X_{i n}, Y_{i n}\right)$, it can always find at least one neighboring pixel $\left(X_{i m}, Y_{i m}\right)$ that satisfies the following required relationships: $\left|X_{i m}-X_{i n}\right| \leq 1$ and $\mid Y_{i m}{ }^{-}$ $Y_{\text {in }} \mid \leq 1$. The recurrence is automatically stopped when there is no new pixel found, and pixels satisfying the above requirement belong to the same white spot.

Based on the sub-sets $\left(L_{i}\right)$ of pixels belonging to individual white spots, the averaged coordinate $\left(X_{a i}, Y_{a i}\right)$ in $L_{i}$ is obtained. $\left(X_{a i}, Y_{a i}\right)$ and $\left(X_{a j}, Y_{a j}\right)$ satisfying $X_{a i}+X_{a j}=$ $L$ are considered as a set of coordinates that correlates to diffraction spots in opposite directions from the same group of parallel lattice planes. The distance $(D$, in the unit of pixiel) between any pair of white spots, i.e., $\left(X_{a i}\right.$ $\left.Y_{a i}\right)$ and $\left(X_{a j}, Y_{a j}\right)$, can be calculated by Equation (1):

$D=\frac{\sqrt{\left(X_{a i}-X_{a j}\right)^{2}+\left(Y_{a i}-Y_{a j}\right)^{2}}}{2}$

Finally, the lattice spacing $(d)$ corresponding to this pair of spots can be obtained based on the equation below:

$d=\frac{m \cdot D}{L}$,

where $L$ is the width (nm) of the FFT image as mentioned before; $m$ is the size $(\mathrm{nm})$ of each pixel in the original dm3 image.

The angle $\theta$ between the planes corresponding to the two pairs of diffraction spots, i.e., $\left(X_{a i 1}, Y_{a i 1}\right),\left(X_{a j 1}, Y_{a j 1}\right)$ and $\left(X_{a i 2}, Y_{a i 2}\right),\left(X_{a j 2}, Y_{a j 2}\right)$, can be obtained by Equation (3):

$\theta=\left|\arctan \left(\frac{X_{a i 1}-X_{a j 1}}{Y_{a i 1}-Y_{a j 1}}\right)-\arctan \left(\frac{X_{a i 2}-X_{a j 2}}{Y_{a i 2}-Y_{a j 2}}\right)\right|$.

We first demonstrate our program for $d$-spacing measurement of a randomly selected HRTEM image of iron oxide nanoparticles, whose composition and structure 

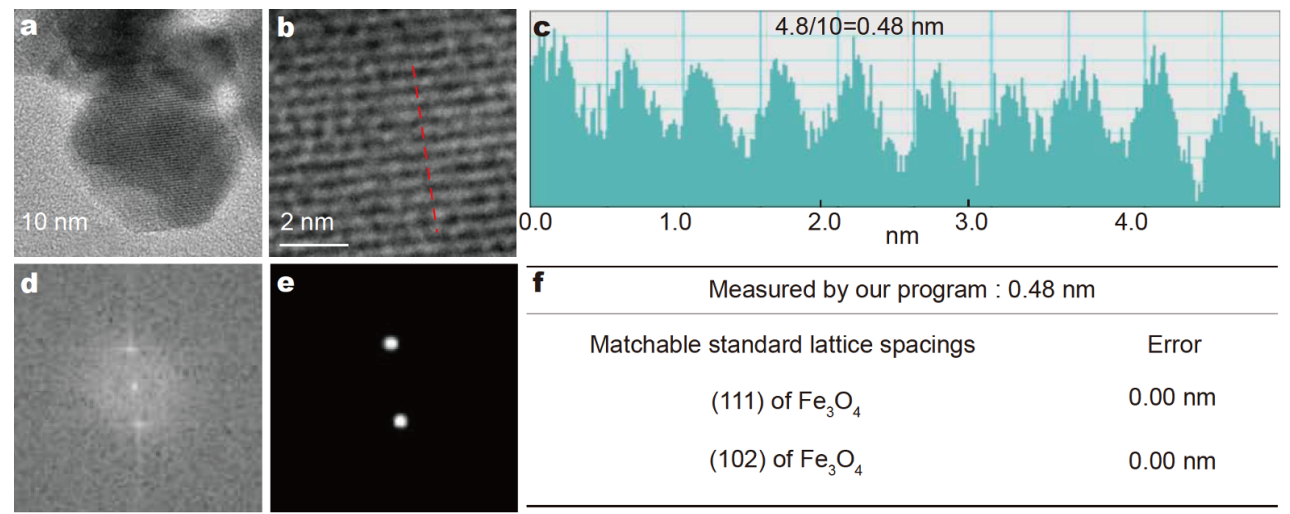

\begin{tabular}{cc}
\hline f & Measured by our program : $0.48 \mathrm{~nm}$ \\
\hline Matchable standard lattice spacings & Error \\
(111) of $\mathrm{Fe}_{3} \mathrm{O}_{4}$ & $0.00 \mathrm{~nm}$ \\
(102) of $\mathrm{Fe}_{3} \mathrm{O}_{4}$ & $0.00 \mathrm{~nm}$ \\
\hline
\end{tabular}

Figure 4 An example of our program applied to analyze a randomly selected HRTEM image of $\mathrm{Fe}_{3} \mathrm{O}_{4}$ particles. (a) $\mathrm{HRTEM}_{\text {image of Fe }} \mathrm{O}_{4}$ particles (b) Zoom-in HRTEM image showing parallel lattice planes. (c) Manual selection and measurement of lattice spacing with Gantan Digital Micrograph software. (d) FFT image of the image in (b). (e) Spots extracted by our program developed from deep learning. (f) Measured lattice spacing in comparison with standard data.

were pre-determined by X-ray diffraction (Fig. S2). Our results showed that the error for spot prediction was always less than $1 \%$. As shown in Fig. $4 \mathrm{a}-\mathrm{c}$, the lattice spacing was first measured manually by averaging the spacing of 10 parallel lattice fringes to be $0.48 \mathrm{~nm}$. By using our program, a value of $0.48 \mathrm{~nm}$ was immediately obtained after inputting the HRTEM image. More importantly, the PDF database is incorporated in our program, and thus after the program determines the lattice spacing, it can spontaneously look for standard lattice spacings that match with our measurement result. As shown in Fig. $4 \mathrm{e}$ and $\mathrm{f}$, the program has identified $\mathrm{Fe}_{3} \mathrm{O}_{4}$ (111) and $\mathrm{Fe}_{3} \mathrm{O}_{4}$ (102) to be the possible planes assignable to the measured lattice spacing. The PDF database used in our program is obtained from The International Centre for Diffraction Data (ICDD, www.icdd.com).

The demonstration of our program to analyze an HRTEM with 2D lattice is provided in Fig. 5. Fig. $5 \mathrm{a}$ can

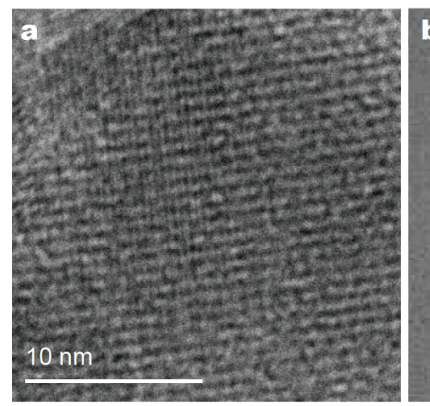

b

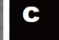

\begin{tabular}{llcc}
\hline d & & Spot 1 & Spot 2 \\
\hline & Measured lattice spacing & $0.13 \mathrm{~nm}$ & $0.24 \mathrm{~nm}$ \\
& Matchable lattice plane & $(-104)$ & $(-112),(311),(222),(021)$ \\
\hline
\end{tabular}

\begin{tabular}{cc}
\hline \hline $\mathbf{e}$ & Inter-plane angle (spots 1 and 2$)$ \\
\hline Determined from FFT & $86.579^{\circ}$ \\
& $64.29^{\circ}$ between $(-104)$ and $(021)$ \\
Standard & $26.99^{\circ}$ between $(-104)$ and $(-112)$ \\
& $85.81^{\circ}$ between $(-104)$ and $(311)$ \\
& $65.16^{\circ}$ between $(-104)$ and $(222)$ \\
\hline
\end{tabular}

Figure 5 An example of our program applied to analyze an HRTEM image with 2D lattice. (a) HRTEM image with 2D lattice. (b) FFT pattern of the image in (a). (c) Spots extracted by our program. (d) Automatically measured lattice spacings, and assigned possible planes for spots 1 and 2 as denoted in (c), respectively. Note that an error of no more than $0.01 \mathrm{~nm}$ is allowed when assigning the standard planes. (e) Comparison between the measured and calculated standard inter-plane angles for planes corresponding to spots 1 and 2. 
produce an FFT image with two pairs of diffraction spots (Fig. 5b). The processed diffraction spots with our program are shown in Fig. $5 \mathrm{c}$. The automatically measured lattice spacings and the matchable crystal planes of $\mathrm{Fe}_{3} \mathrm{O}_{4}$ are shown in Fig. 5d. In addition, we also include a function of calculating inter-plane angles of standard crystal structures in our program. It can be seen that when spot 1 is assigned to $(-104)$ plane and spot 2 is assigned to (311) plane, the inter-plane angle determined from the FFT is consistent with the standard one. Analyses on HRTEM images with polycrystalline domains or multiple nanoparticles are shown in Figs S3 and S4, respectively.

In summary, an efficient TEM image processing method has been developed in this work to achieve objective, rapid and automatic measurement of lattice spacings and inter-plane angles in HRTEM images. This method uses the U-net-based neural network for the identification and extraction of central symmetric bright spots in an FFT image. In the subsequent processing, a special measurement method was developed to locate the bright spots in the FFT image and calculate the lattice spacings and inter-plane angles according to the coordinates of the bright spots. The calculated spacings were further compared with the standard database to search for matchable crystalline planes. Our method holds great promise in assisting the efficient structural analysis of crystalline materials.

Received 20 January 2020; accepted 22 April 2020;

published online 9 July 2020

1 Madsen J, Liu P, Kling J, et al. A deep learning approach to identify local structures in atomic-resolution transmission electron microscopy images. Adv Theor Simul, 2018, 1: 1800037

2 Hussaini Z, Lin PA, Natarajan B, et al. Determination of atomic positions from time resolved high resolution transmission electron microscopy images. Ultramicroscopy, 2018, 186: 139-145

3 Guven G, Oktay AB. Nanoparticle detection from TEM images with deep learning. In: Proceedings of the Signal processing Communications Applications Conference. 2018

4 de Ruijter WJ, Sharma R, McCartney MR, et al. Measurement of lattice-fringe vectors from digital HREM images: Experimental precision. Ultramicroscopy, 1995, 57: 409-422

5 Oshida K, Endo M, Nakajima T, et al. Image analysis of TEM pictures of fluorine-intercalated graphite fibers. J Mater Res, 1993, 8: $512-522$

6 Murugan P. Feed forward and backward run in deep convolution neural network. In: Proceedings of the 20th International Conference on Computer Vision and Image Processing. 2017

7 LeCun Y, Bengio Y, Hinton G. Deep learning. Nature, 2015, 521: 436-444

8 Goodfellow IJ, Pouget-Abadie J, Mirza M, et al. Generative adversarial nets. Neural information processing systems. 2014, $2672-$
2680

9 Koutnik J, Greff K, Gomez F, Schmidhuber J. A clockwork RNN. In: Proceedings of the International Conference on Machine Learning. 2014, 1863-1871

10 Ronneberger O, Fischer P, Brox T, et al. U-net: Convolutional networks for biomedical image segmentation. Medical Image Computing and Computer Assisted Intervention. 2015, 234-241

11 Long J, Shelhamer E, Darrell T, et al. Fully convolutional networks for semantic segmentation. Computer Vision and Pattern Recognition. 2015, 3431-3440

12 Giusti A, Cireşan DC, Masci J, et al. Fast image scanning with deep max-pooling convolutional neural networks. In: Proceedings of the International Conference on Image Processing. 2013, 4034-4038

13 Hadji I, Wildes RP. What do we understand about convolutional networks. arXiv: 1803.08834

Acknowledgements This work was supported by the National Key Research and Development Program of China (2017YFA0104302), and the National Natural Science Foundation of China (61821002, 51832001 and 31800843).

Author contributions $\mathrm{Gu} N$ conceived the idea. Zhu X and Zhang Z designed and completed the measurement method. Mao Y prepared the materials and performed the TEM and XRD measurements. Zhu X, Huang $\mathrm{X}$ and Li Y wrote and revised the paper. All authors contributed to the general discussion.

Conflict of interest The authors declare that they have no conflict of interest.

Supplementary information Experimental details and supporting information are available in the online version of the paper.

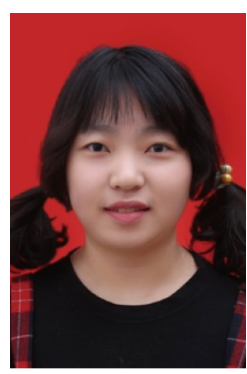

Xiaoyang Zhu received her bachelor's degree in 2019 at Southeast University (SEU). Her current research interest is the combination of artificial intelligence and materials science.

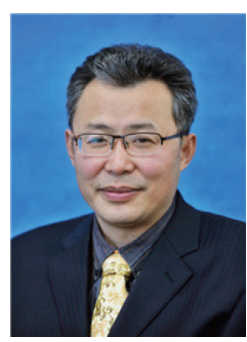

Ning Gu received his $\mathrm{PhD}$ degree in biomedical engineering from the Department of Biomedical Engineering, Southeast University, Nanjing, China, in 1996. Currently he is a Cheung Kong Scholar Chair Professor at the School of Biological Science and Medical Engineering, Southeast University. 


\section{深度学习用于HRTEM图像晶格间距的快速自动} 测量

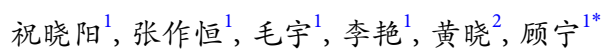

摘要 在图像处理与分析领域, 深度学习发挥着日渐重要的作用. 我们将深度学习应用于高分辨率透射电子显微镜(HRTEM)图像晶 面间距的自动快速测量中, 通过对随机样本数据训练得到具有 U 型 结构的神经网络, 开发了一种新的图像处理方法. 本方法能够自动 提取快速傅里叶变换(FFT) 图像中的衍射斑点, 进一步在计算机视 觉技术的协助下, 可以自动计算与FFT图像中被识别的衍射点相对 应的晶格间距, 并与标准晶体结构数据进行比较. 以 $\mathrm{Fe}_{3} \mathrm{O}_{4}$ 纳米粒子 的HRTEM图像为例, 用本方法进行自动测量的晶格间距与手动测 量的晶格间距相比, 误差小于 $1 \%$. 我们的工作证明了深度学习技术 在协助晶体材料发展方面的巨大潜力. 